\title{
Naltrexone and Scopolamine Rapidly Reduce Symptoms of Major Depressive Disorder (MDD): A Double Blinded Randomized Controlled Pilot Study
}

\author{
N. Taub \\ The Charlotte Ketamine Center, Charlotte, NC, USA \\ Email: nealtaub@gmail.com
}

How to cite this paper: Taub, N. (2019). Naltrexone and Scopolamine Rapidly Reduce Symptoms of Major Depressive Disorder (MDD): A Double Blinded Randomized Controlled Pilot Study. Open Journal of Depression, $8,1-4$.

https://doi.org/10.4236/ojd.2019.81001

Received: December 3, 2018

Accepted: January 8, 2019

Published: January 11, 2019

Copyright $\odot 2019$ by author(s) and Scientific Research Publishing Inc. This work is licensed under the Creative Commons Attribution International License (CC BY 4.0).

http://creativecommons.org/licenses/by/4.0/

\begin{abstract}
Background: Scopolamine and naltrexone are FDA approved medications that have been utilized off label for the indication of major depressive disorder (MDD). We examined the efficacy of the 2 medications in combination for major depression. Methods: 14 adults ( 7 female and 7 males) with current major depressive disorder were randomized by coin flip to $0.15 \mathrm{mg}$ BID of scopolamine and $1 \mathrm{mg}$ BID naltrexone or placebo for a total of 4 weeks. Modified MDRS questionnaires were utilized to monitor progress in depression symptomatology. The placebo patients were crossed over to the active medications subsequent to the 4 week trial period. Results: 2 subjects dropped out of the trial as a result of side effects. A total of 12 subjects completed the trial. The average reduction in Modified MDRS scores over the 4 weeks for the active medications was 12.5. The average reduction noted in control patients was 3.5. This difference was statistically significant with $P=0.03$, less than 0.05 for a greater than $95 \%$ confidence interval utilizing Student's T test analysis. Of the 4 patients that crossed over to the active medications three additional patients noted a partial or complete response to the medications with an average reduction and Modified MDRS scores of 8 . No washout periods were utilized before the trial or for the crossover period. Conclusion: The combination of scopolamine and naltrexone demonstrated significant benefit for MDD.
\end{abstract}

\section{Keywords}

Depression, MDD, Scopolamine, Naltrexone

\section{Introduction}

Depression is currently a silent pandemic, and according to the World Health 
Organization accounts for more disability worldwide than any other medical problem. Most individuals with depression do not receive treatment. Those that do are treated with many modalities with only fair efficacy. More than a third of treated patients do not achieve remission with current treatments. A number of recent studies have utilized antidepressant medications in combination and generally synergy is noted with increased levels of response and remission reported, often with no increased adverse effect burden.

Naltrexone is an opioid antagonist FDA approved for treatment of ethanol use disorder as well as opioid use disorder. Naltrexone Hydrochloride is a competitive opioid antagonist at both mu and delta opioid receptors. An oral dose of 50 - $100 \mathrm{mg}$ can reverse opioid overdoses. Paradoxically, ultra-low dose naltrexone (less than $1 \mathrm{mcg}$ ) enhances the effects of opioid agonists. Naltrexone binds to the C-terminal pentapeptide of the scaffolding protein filamin A with strong avidity which may prevent or reverse a change in G-protein signaling in G-coupled receptor systems, such as the mu opioid receptor after prolonged stimulation by an agonist. Low dose naltrexone has additionally been anecdotally found to be helpful in many disorders, including fibromyalgia, which has symptom overlap with depression. Naltrexone has recently been noted in a pilot study to be effective in treating depression relapse in patients utilizing dopaminergic antidepressants (Mischoulon et al., 2017).

Scopolamine has been demonstrated to be a rapid acting antidepressant administered both as an IV infusion as well as dosed orally. Interest in the muscarinic cholinergic system in mood disorders stemmed initially from evidence suggesting hypersensitivity of the cholinergic system plays a role in the pathophysiology of depression. Researchers have shown increasing cholinergic activity exacerbates depressive symptoms. Two studies have demonstrated that scopolamine administered both IV and orally result in rapid remission from major depression in a majority of patients (Han \& Pae, 2013; Drevets et al., 2013; Furey \& Drevets, 2016; Khajavi et al., 2012). The principle investigator has utilized scopolamine in the treatment of depression and anxiety IV and orally with a high level of efficacy and minimal side effects.

We carried out a study utilizing the oral route of administration in order to maximize convenience and cost effectiveness. The $1 \mathrm{mg}$ B.I.D. naltrexone dose and $0.15 \mathrm{mg}$ B.I.D. dose of scopolamine combination was chosen based on our clinical experience with the medications, and our desire to maximize both efficacy and tolerability.

\section{Methods}

The study was approved by IntegReview IRB, informed consent was obtained, and the study was registered at Clinicaltrials.gov: \#NCT03386448. Charlotte North Carolina area men and women with MDD were recruited by Internet and billboard advertisement.

Inclusion criteria included: Age 18 - 65; written informed consent; meeting clinical diagnosis of MDD through interview and also through Modified MDRS 
score greater than or equal to 20 . All subjects had previously utilized at least one other antidepressant medication; accepted subjects had no changes in current medications for the last 4 weeks. Average duration of depression was 34 months.

Exclusion criteria included: suicidal ideation; severe kidney or liver disease; recent change in medication; allergy or hypersensitivity to naltrexone or scopolamine; use of opioids or MAO inhibitors; glaucoma; schizophrenia; pregnancy or lactation.

The patients were randomized into an active medication group and a control group (7 patients each group). The patients began the medication and were reevaluated weekly. At the four week point, the medication codes were identified. All control medication patients elected to begin active medication and 5/6 in the active group continued the active medications. In the active group bupropion was added to the regime of 3 patients with no response or only partial responses, and the medication doses were increased to a maximum of $0.5 \mathrm{mg}$ of scopolamine and $3 \mathrm{mg}$ daily of naltrexone in non-responders.

Subsequently 3 patients from the control group noted at least a partial response with the active medications. In the active group, of the two patients that did not initially respond, no significant improvement was noted later.

\section{Results}

2 subjects dropped out of the trial as a result of side effects. A total of 12 subjects completed the trial. The average reduction in Modified MDRS scores over the 4 weeks for the active medications was 12.5. The average reduction noted in control patients was 3.5 (See Table 1). This difference was statistically significant with $P=0.03$, less than 0.05 for a greater than $95 \%$ confidence interval utilizing Student's T test. Of the 4 patient's that crossed over to the active medications three additional patients noted a partial or complete response to the medications with an average reduction and MDRS scores of 8 .

\section{Discussion}

Depression is an extremely common and debilitating condition and is responsible for significant mortality and morbidity worldwide. Many patients with depression do not receive any treatment, and a large percentage of treated patients do not significantly improve. Scopolamine and naltrexone have both been noted in studies to significantly augment other medications. The remission rates were comparable to those noted in this study (Han \& Pae, 2013; Drevets et al., 2013; Furey \& Drevets, 2016; Khajavi et al., 2012).

Table 1. Average MDRS changes with active treatment vs. controls.

\begin{tabular}{ccc}
\hline Group & Average MDRS Change & Significance \\
\hline Active Treatment Group & 12.5 & Yes: $P=0.03$ \\
Control Group & 3.5 & \\
\hline
\end{tabular}


In this pilot study we demonstrated a rapid and robust improvement in depression symptoms with the combination of scopolamine and naltrexone.

\section{Conclusion}

This pilot study demonstrated a robust and rapid reduction in a majority of depressed subjects with a tolerable side effect profile. This combination has significant potential as an adjunct treatment for treatment resistant MDD or potentially as a first line treatment.

\section{Conflicts of Interest}

The author declares no conflicts of interest regarding the publication of this paper.

\section{References}

Drevets, W. C., Zarate Jr., C. A., \& Furey, M. L. (2013) Antidepressant Effects of the Muscarinic Cholinergic Receptor Antagonist Scopolamine: A Review. Biological Psychiatry, 73, 1156-1163.

Furey, M. L., \& Drevets, W. C. (2006) Antidepressant Efficacy of the Antimuscarinic Drug Scopolamine. Archives of General Psychiatry, 63, 1121-1129. https://doi.org/10.1001/archpsyc.63.10.1121

Han, C., \& Pae, C.-U. (2013) Oral Scopolamine Augmentation for Major Depression. EXpert Review of Neurotherapeutics, 13, 19-21. https://doi.org/10.1586/ern.12.150

Khajavi, D. et al. (2012) Oral Scopolamine Augmentation in Moderate to Severe Major Depressive Disorder: A Randomized, Double Blinded, Placebo-Controlled Study. Journal of Clinical Psychiatry, 73, 1428-1433. https://doi.org/10.4088/JCP.12m07706

Mischoulon, D. et al. (2017) Randomized, Proof of Concept Trial of Low-Dose Naltrexone for Patients with Breakthrough Symptoms of Major Depressive Disorder on Antidepressants. Journal of Affective Disorders, 208, 6-14. 